\title{
Mechanical properties of hypothetical graphene foams: Giant Schwarzites
}

\author{
David Miller ${ }^{\mathrm{a}}$, Mauricio Terrones ${ }^{\mathrm{b}}$, Humberto Terrones ${ }^{\mathrm{a}, *}$ \\ ${ }^{a}$ Department of Physics, Applied Physics, and Astronomy, Rensselaer Polytechnic Institute, Troy, NY \\ 12180-3590, USA. \\ ${ }^{b}$ Department of Physics and Center for 2-D and Layered Materials, Department of Chemistry, Department of \\ Materials Science and Engineering, The Pennsylvannia State University, University Park, PA 16802-6300, USA
}

\begin{abstract}
The mechanical properties of four different families of ordered porous graphene or giant Schwarzites, up to 12,288 atoms per cubic cell, were studied theoretically in order to shed light on the properties of newly synthesized graphene-like foams. It is shown that as the Schwarzite grows, the structure becomes flatter and not only more energetically stable, but also more elastically stable, thus opening the possibility of being synthesized in the near future. The mechanical properties such as bulk modulus, Young's modulus, and Poisson's ratio have been calculated with first principles for the smaller cells and with empirical methods for the larger cells. The bulk and Young moduli decrease as the structures grow. The "P" and the "I-WP" geometries favor smaller values of Poisson's ratio, likely to be synthesized experimentally. For the larger gyroid "G" and "D" cases, elastic instabilities appear, and these can be alleviated by breaking the symmetry of the associated space group. In addition, ripples in the graphene sheet stabilize the giant " $D$ " family as the crystal cell dimensions increase. Finally, based on density functional theory calculations, the electronic properties of the high genus I-WP were examined for the first time finding semiconducting, semimetallic, and metallic behavior.
\end{abstract}

\section{Introduction}

Since the proposition of negative Gaussian curvature in graphitic systems through the introduction of carbon rings with more than six atoms, it was recognized the possibility of synthesizing negatively curved $\mathrm{sp}^{2}$-hybridized carbon, named Schwarzites, which exhibit topologies similar to those found in triply periodic minimal surfaces (TPMS) [1-3]. At that time, calculations showed the great stability and the diverse electronic properties of Schwarzites being insulators, semiconductors or metals [4]. The recent exciting properties found in experimentally synthesized graphene foams have brought back the challenging task of finding three dimensional graphene with similar features to Schwarzites [5].

${ }^{*}$ Corresponding author. Tel: 518276 2932. E-mail: terroh@rpi.edu (Humberto Terrones). 
TPMS are a class of minimal surfaces, corresponding to zero mean curvature at every point, that are also periodic in three dimensions, and thus are crystalline. These surfaces all display nonpositive Gaussian curvature since the two principal curvatures are opposite in sign and equal in magnitude, and exhibit saddle shapes at every point. In this paper, we examined four families of TPMS deocrated with graphene, the Primitive (P), Diamond (D), Gyroid (G) and I-graph Wrapped Package graph (I-WP) surfaces (see Fig. 1). The P (Pm $\overline{3} m-\operatorname{Im} \overline{3} m$ space groups) and D (Fd $\overline{3} m-P n \overline{3} m$ space groups) surfaces were first proposed by Hermann Schwarz [2, 6] and the G ( $I a \overline{3} d$ space group) and I-WP ( $P m \overline{3} m-\operatorname{Im} \overline{3} m$ space groups) were later proposed by Alan Shoen [7]. Small and giant Schwarzites exhibit similar topologies and space groups as the TPMS, however, giant Schwarzites such as the ones we are studying here, are more graphenic in the sense that the ratio of hexagons to rings with more than six atoms, is very high, so are locally flat. Very little has been discussed about the properties of these giant Schwarzites, mainly due to the lack of experimental evidence and the difficulties to generate and deal with large number of atoms in theoretical calculations. In this paper we generate and study the mechanical properties of giant Schwarzites up to 12288 atoms per cubic cell. These calculations might help in understanding the newly synthesized graphene foams $[5,8]$ and to predict novel graphenic arrangements in three dimensions.

One of the most exciting properties of graphene, a single layer sheet of graphite, is its unique linear energy dispersion close to the $\mathrm{K}$ points in the Brillouin zone forming Dirac cones, in which electrons behave as Dirac Fermions [9-11]. This behavior has been recently found theoretically in a gyroid "G" Schwarzite with 1536 atoms in the cubic cell, exhibiting hyper Dirac cones in the valence and conduction bands which can be shifted to the Fermi level by lithium doping [12]. In this context, electronic properties of smaller Schwarzites have been studied and no Dirac Fermion behavior has been found. However, they can be metals, semimetals, semiconductors or insulators depending on their geometry and their size [4]. This diversity in electronic behavior with $\mathrm{sp}^{2}$-hybridized carbon atoms, in addition to the newly observed Poisson ratio close to zero in a graphene foam [5], justifies the current study on giant Schwarzite systems not reported hitherto.

In this paper, we computed 35 Schwarzites based on D, G, P and I-WP TPMS using density 
$\mathrm{a}$

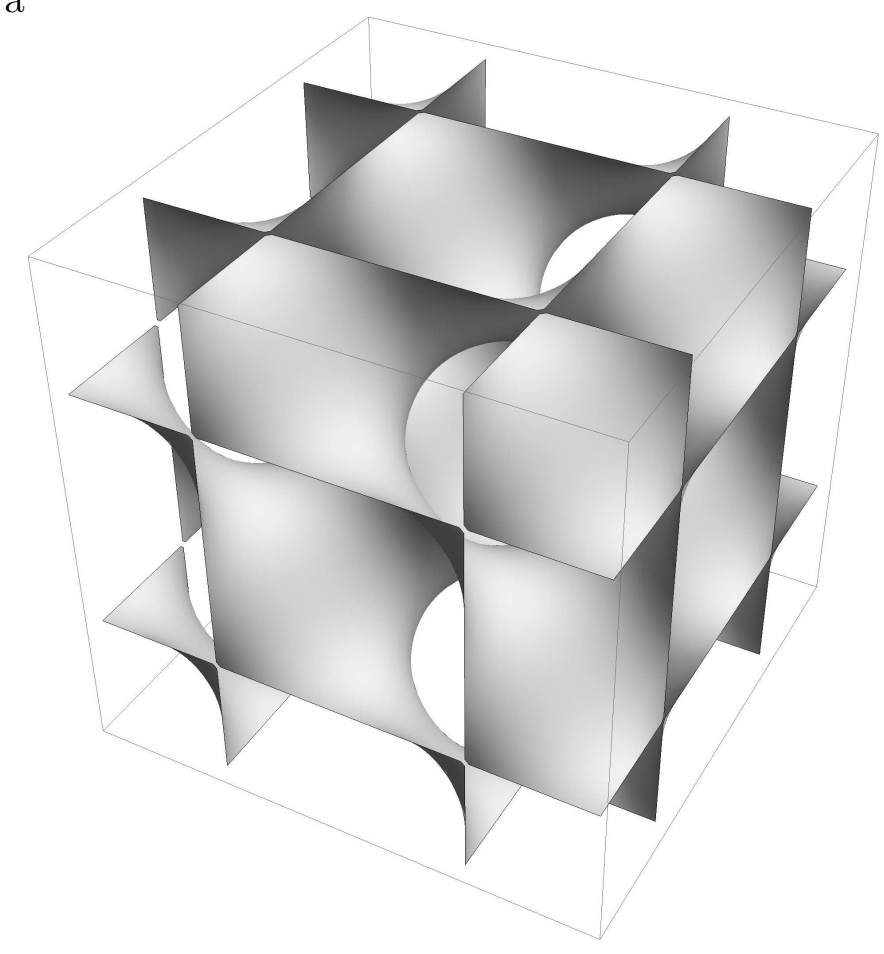

c

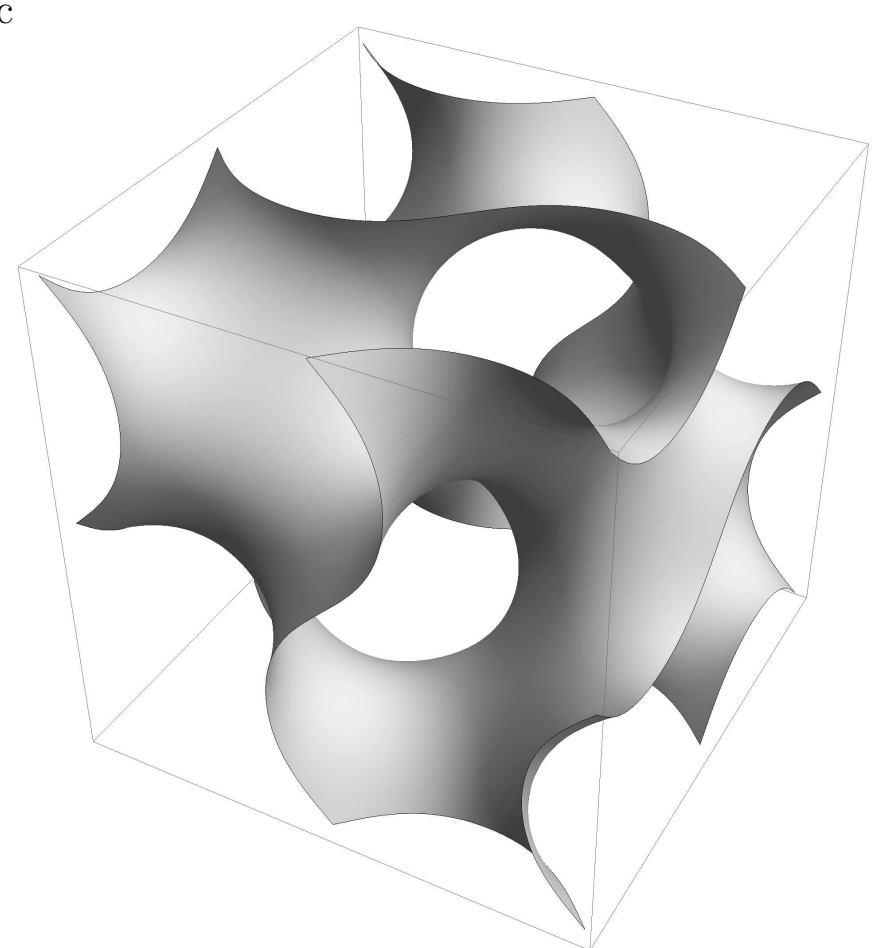

b

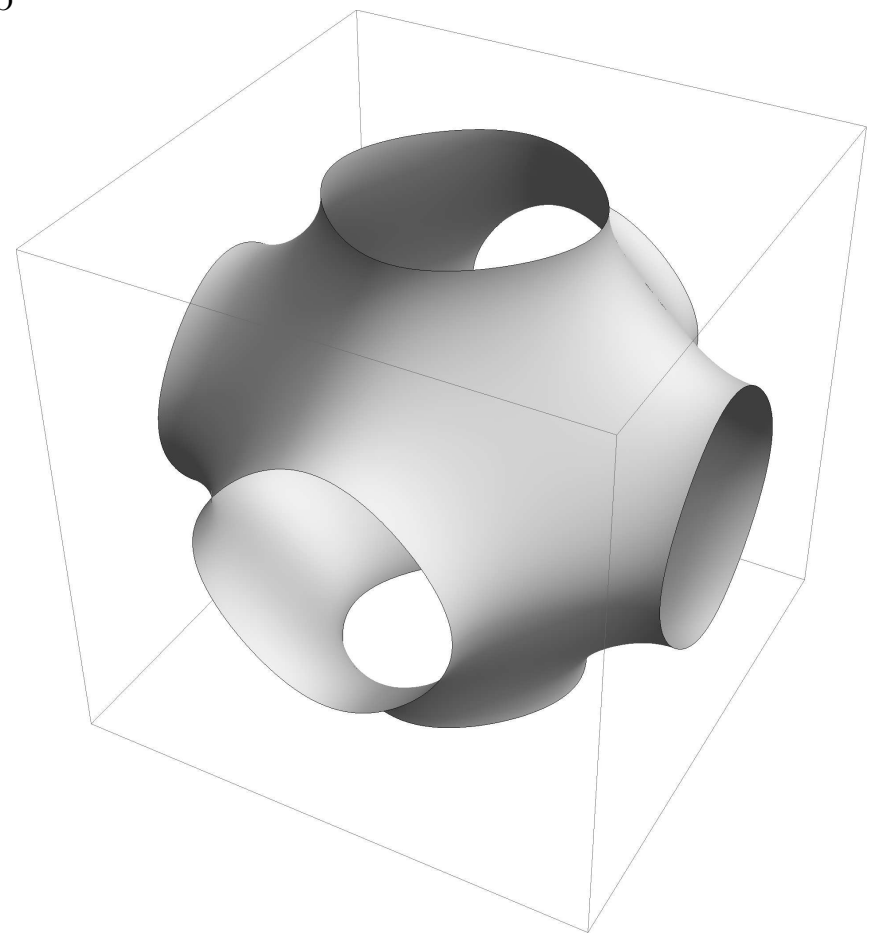

d

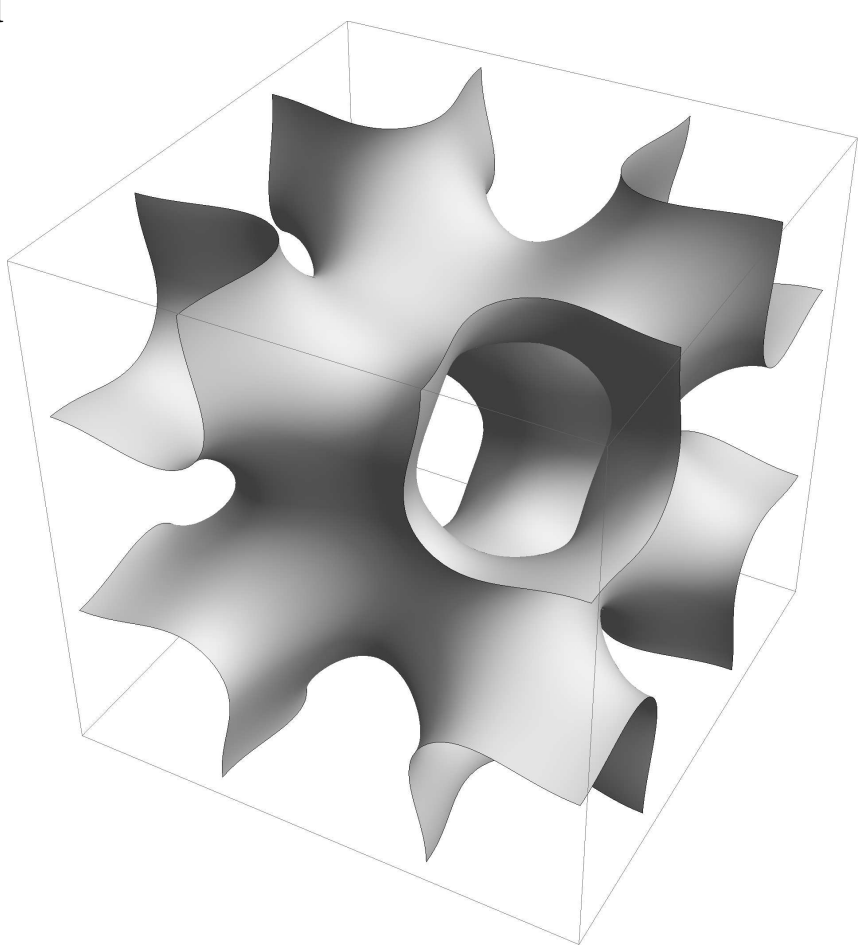

Figure 1: A single cubic cell of the (a) D, (b) P, (c) G, and (d) I-WP minimal surfaces.

functional theory (DFT) and empirical methods: The energetics, elastic stability and mechanical properties such as Young modulus, bulk modulus and Poisson ratio were calculated. Emphasis has been put on giant Schwarzites that have not been studied before. We also calculated for the first 
time, using DFT, the electronic structure for the smallest cases of the I-WP structures finding that they exhibit semi-metallic, metallic and semi-conducting behavior depending on size. We found that the giant P and I-WP Schwarzites can be grown preserving the topology and symmetry (space group), and this is not the case for the giant G and D Schwarzites that require the space group symmetry to be broken or the presence of ripples to be elastically stable. With the term "grow" we mean that the structure can be "inflated" by the addition of hexagonal rings, thus preserving the number of rings with more than six carbon atoms, such as octagonal carbon rings. In other words, the Schwarzites we have studied are formed by varying the number of hexagons with a fixed number of octagons. We found that the $\mathrm{P}$ giant Schwarzites exhibit the lowest average Poisson ratios around 0.183. If defects, such as ripples, are considered, it is possible to lower the Poisson ratios as we will see for the D giant Schwarzites.

\section{Methods}

\subsection{Generation of Schwarzites with Surface Evolver}

In a decorated TPMS using only hexagonal and octagonal rings, it is possible to derive the number of octagons that should be present through the Gauss-Bonnet theorem as shown in Eq. 1 where "g" is the genus per primitive cell and $N_{5}, N_{7}$ and $N_{8}$ are the number of pentagons, heptagons and octagons, respectively, present in the Schwarzite [2]. It can be easily verified that the $\mathrm{P}, \mathrm{D}$, and $\mathrm{G}$ surfaces with genus equal to 3 will have 12 octagons in the primitive cell and the I-WP surface, with genus per primitive cell equal to 4, will have 18 octagons in the primitive cell. Given an initial continuous surface patch for each surface, the Surface Evolver code [13] was used to calculate each decorated surface with carbon atoms associated to a TPMS, and then to increase the size of the structure. For the initial patch for each surface the octagonal rings are located in the region with the greatest magnitude in Gaussian curvature. For the I-WP, the location of some octagons was also determined to preserve the symmetry of the surface. Surface Evolver calculates the local minimum of a specified energy functional iteratively using a gradient descent method [13]. For our purposes, that energy was calculated by minimizing a weighted sum of the surface area, the squared mean curvature, and the deviation of the atomic separation. Finally, the center 
of each facet in Surface Evolver is used as the location of an atom on the calculated surface. See Fig. 2 as an example of an overlay of the decorated surface on the calculated surface.

$$
N_{5}-N_{7}-2 N_{8}=12(1-g)
$$

Through the use of Surface Evolver we have generated all the initial coordinates for each of the four families studied. Each family contains two sub-families, the normal calculated surface and a second set referred as rotated structures. In these rotated structures, the octagonal rings are rotated by $\pi / 4$ radians which results in a rotation of the hexagonal rings by $\pi / 3$ radians as well as a change in the number of hexagonal rings. Each calculated Schwarzite is named using the following convention. First the abbreviated letter of the surface (P,D,G or I-WP), next an " 8 " referring to the introduction of octagonal rings, followed by the minimum number of hexagonal rings separating two octagonal rings. Lastly, "Rot" is appended in the case of the rotated subfamily (ex. P8-5 or D8-11Rot). Figure 3 shows the difference between the P8-1 structure and the associated rotated P8-2Rot structure.

\subsection{Computer Simulations}

The elastic properties of the porous graphene have been calculated considering different cubic cells using the Brenner reactive empirical bond order (REBO) potential [14] with the code GULP (general utility lattice program) [15]. For the smallest structures, a first principles plane wave DFT approach coded in CASTEP [16] has been used, finding a good agreement between the mechanical properties of GULP and CASTEP. For the first principles calculations, cubic cells were considered under the Local Density Approximation (LDA) using the Ceperly-Alder-Perdew and Zunger (CA-PZ) functional [17, 18] with variable Monkhorst-Pack K-points, depending on the size $(3 \times 3 \times 3$ for the I-WP cases), and a plane waves cut off of $500 \mathrm{eV}$ with a norm-conserving pseudopotential. All the structures were relaxed, including the cells, until the forces became smaller than $0.05 \mathrm{eV} / \AA$ and with self-consistent energy tolerances less than $2 \times 10^{-5} \mathrm{eV} /$ atom. For consistency, all the values of the mechanical properties given in the tables of this paper have been calculated with GULP. 
a

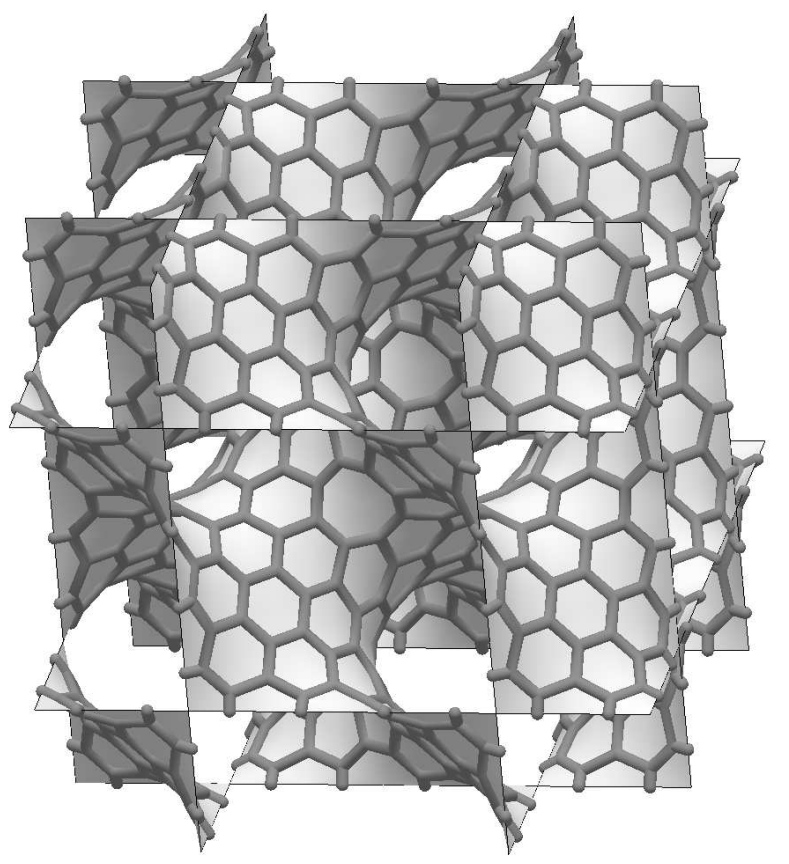

$\mathrm{C}$

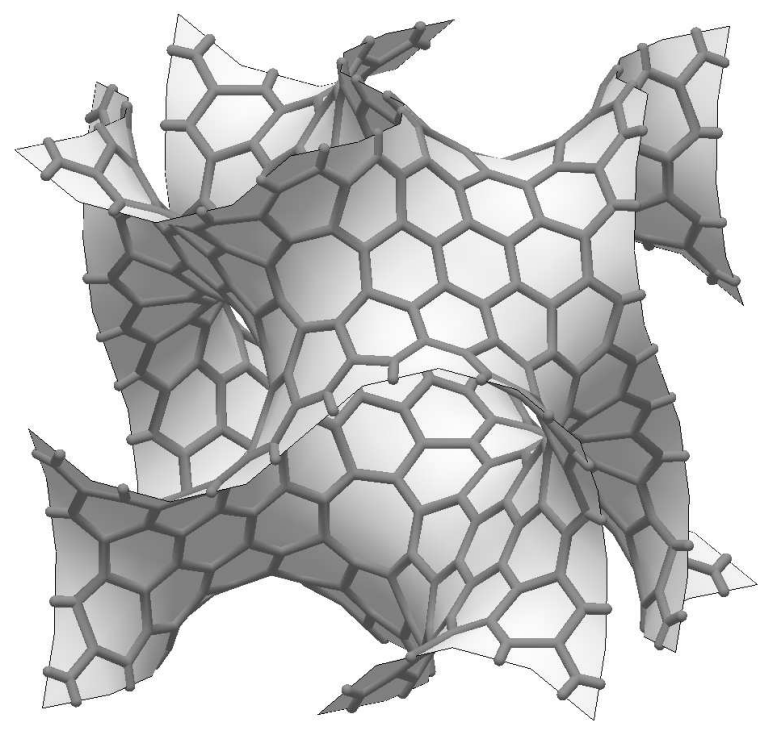

$\mathrm{b}$

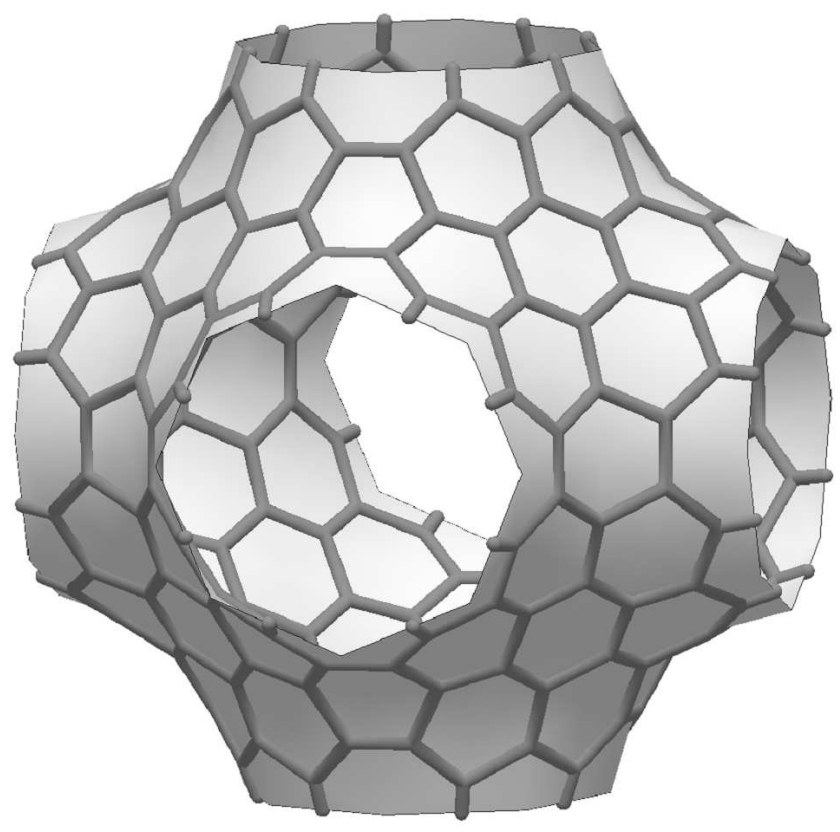

d

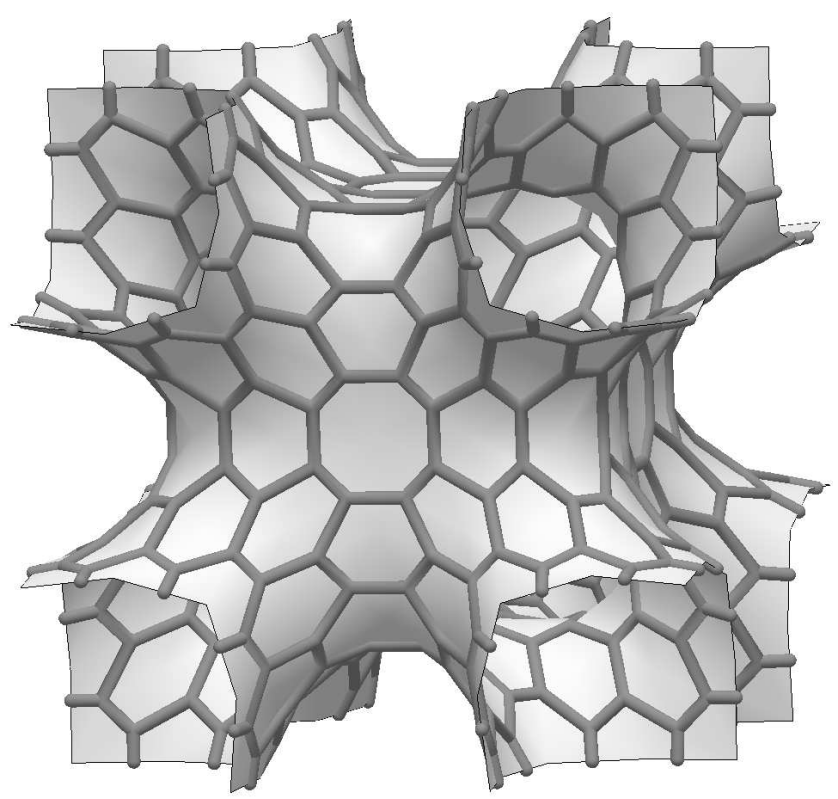

Figure 2: Overlay of the decorated structures on the calculated surfaces using Surface Evolver. All structures shown are of the 8-1 variety. Cubic cells are displayed for (a) the D8-1, (b) the P8-1, (c) the G8-1, and (d) the I-WP8-1. 
a

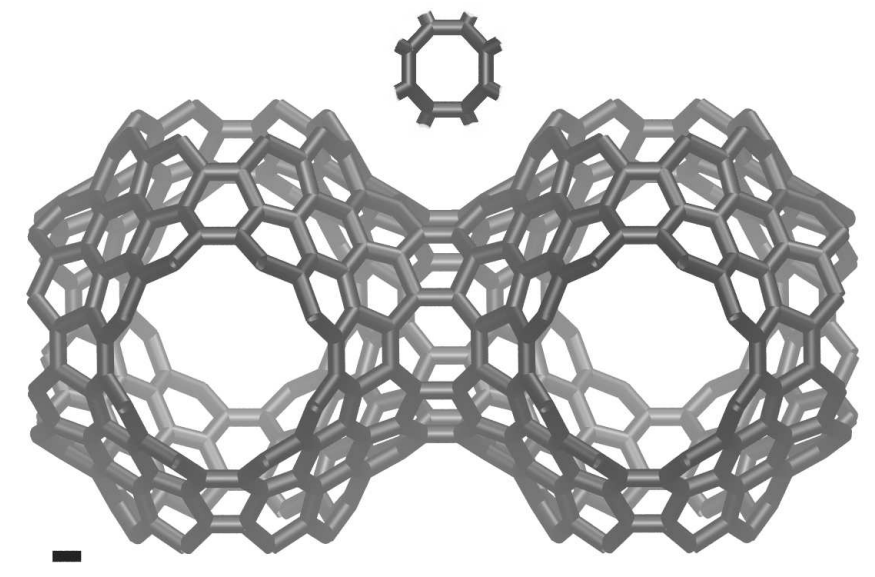

$\mathrm{b}$

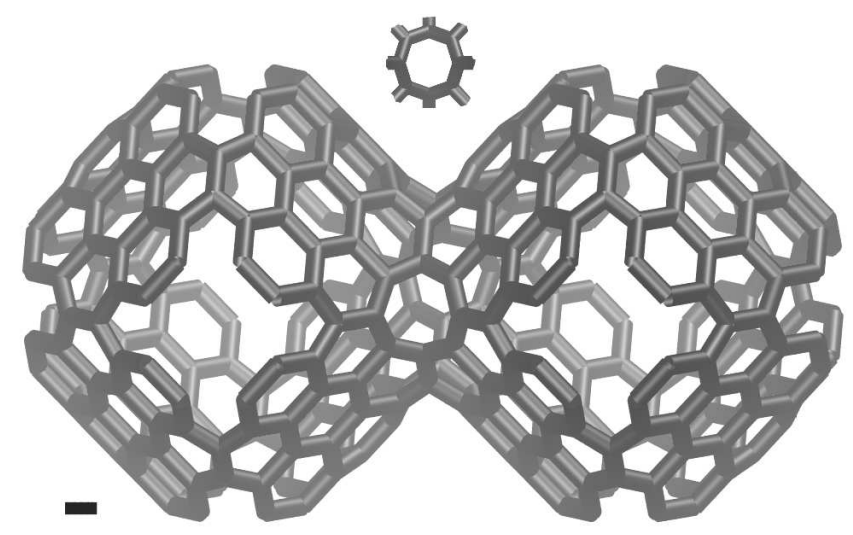

Figure 3: Molecular models of: (a) the decorated P8-1 and (b) the P8-2Rot structures. Each structure is displayed as two cubic cell side by side. The octagonal rings in the P8-2Rot have been rotated by $\pi / 4$ radians and the hexagonal rings have been rotated by $\pi / 3$ radians. Pictured above each structure is the orientation of the octagonal rings. The P8-2Rot has only 144 atoms per cubic cell compared to the P8-1 which has 192 atoms per cubic cell. The scale bar in the bottom left corner of each represents $1 \AA$.

\section{Results}

\subsection{Energetics}

The calculated energetics using the REBO potential (energy per/atom relative to graphene) for all structures examined are shown in Fig. 4. The results obtained for the smaller structures examined from the P, D, and $\mathrm{G}$ families agree relatively well with previous calculations reported in the literature $[4,19]$, but there are no values available to compare for our giant cases up to 12,288 atoms per cubic cell. Of the four families analyzed, the $\mathrm{P}$ family most closely approaches the energy per atom of graphene as increasing the lattice parameter. The I-WP family also approaches low energy values similar to graphene but has much higher energies for the smallest cells. While the G family approaches energetics similar to graphene, structures from this family also display elastic instabilities for larger sizes which do not satisfy the Born criteria [20] (see below). The G8-7 Schwarzite was elastically unstable when symmetrically constrained following the $I a \overline{3} d$ space group. This structure and the G8-23Rot were instead calculated without constraint to symmetry in order to find a more elastically stable structure. We believe that the instability of the giant Gyroids come from the fact that the G TPMS does not contain straight lines and graphene prefers to keep its flatness by possessing straight lines. The D surface family is interesting as the energies do not change much as the size of the cell grows. The addition of hexagons to this family of Schwarzites 
Energetics for Schwarizites compared to graphene

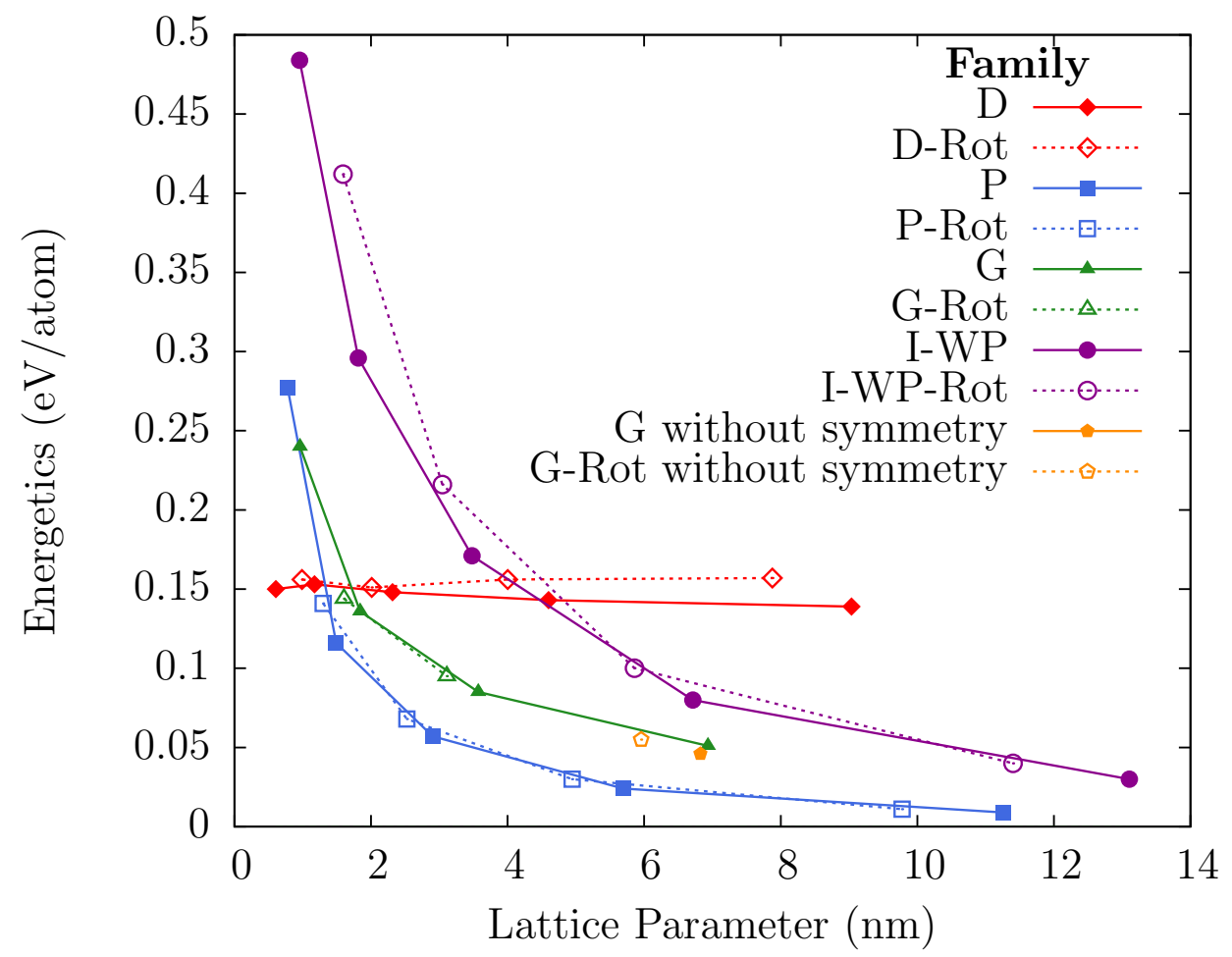

Figure 4: The energetics of Schwarzites relative to graphene. A value of $0 \mathrm{eV}$ per atom is the energetics of graphene calculated using the Brenner empirical potential.

seems to have no effect on lowering the energetics towards that of graphene. In addition, the largest of the D Schwarzites (D8-15 and D8-23Rot) both display ripples as we will discuss latter in detail.

Of the four families of Schwarzites examined here, the P and I-WP families look the most promising to be synthesized since they display energetics that approach that of single layer graphene while remaining elastically stable. Also, from the relaxed structures, there are large patches on the largest structures that appear to closely resemble flat graphene layers. The P8-15 displays flat hexagonal regions that are connected at the octagonal sites (see Fig. 5). The I-WP8-15, with more octagons per cubic cell than the $\mathrm{P}$ family, displays quadrilateral regions that are also flat (see Fig. $6)$.

\subsection{Elastic properties}

For all structures with cubic symmetry the elasticity tensor contains only three unique values, $c_{11}, c_{12}$, and $c_{44}$ (see Table 1). The same holds for the compliance tensor, which is the 
$\mathrm{a}$

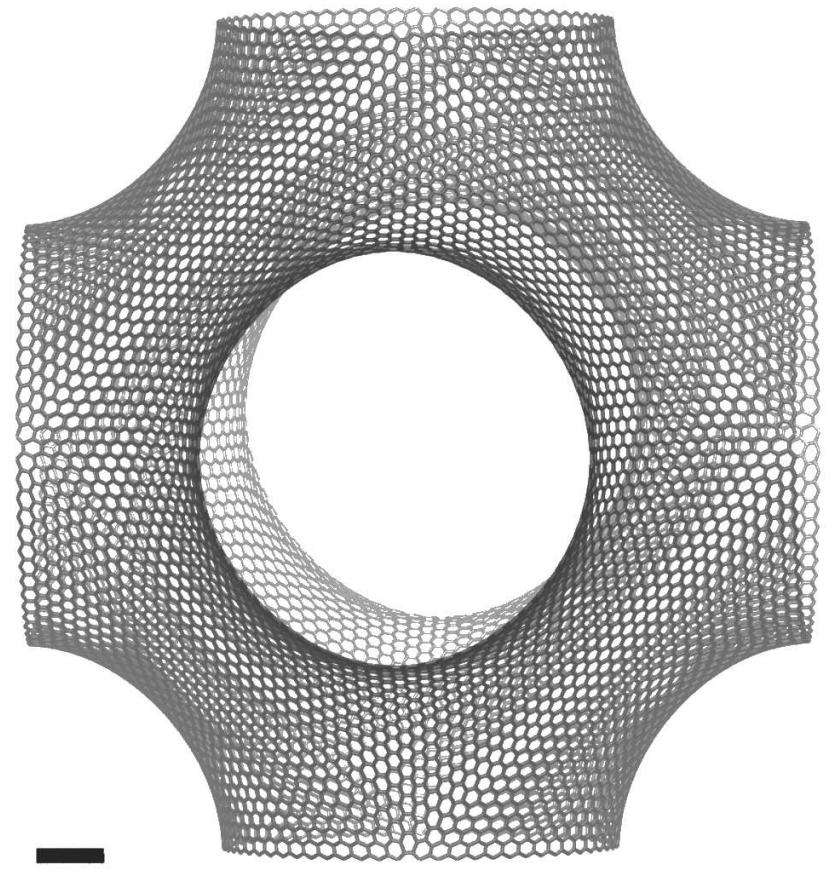

$\mathrm{b}$

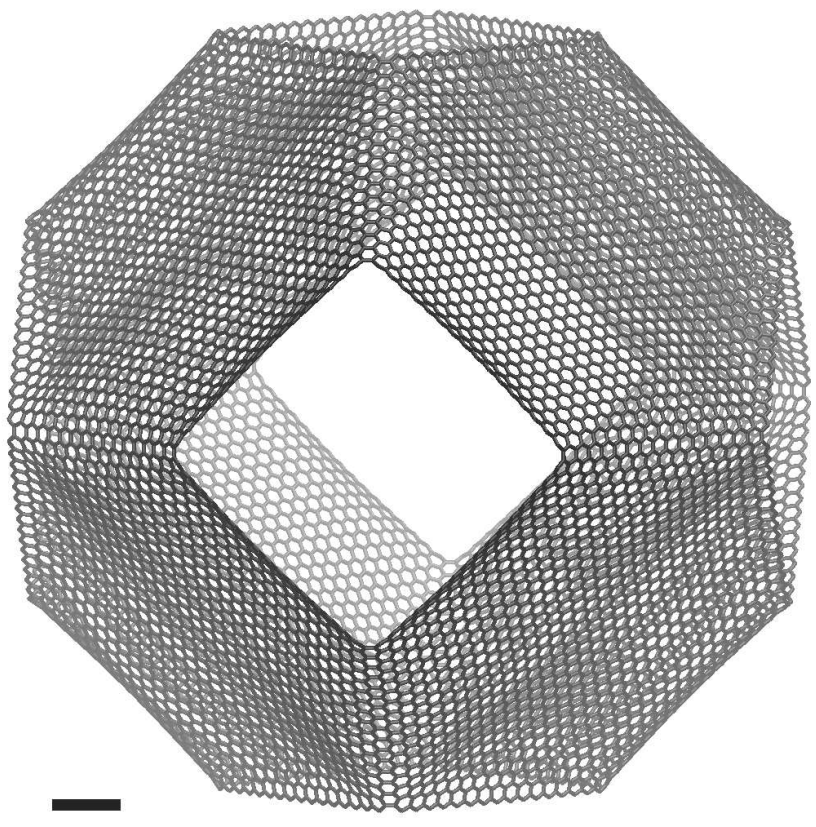

Figure 5: A single cubic cell of the P8-15 structure (a) before relaxation and (b) after relaxation. The hexagonal regions with low curvature are now even flatter as they become more graphenic. Of all the structures examined in this paper, this structure has energetics closest to graphene. The scale bar in the lower left corner is for $10 \AA$.

$\mathrm{a}$

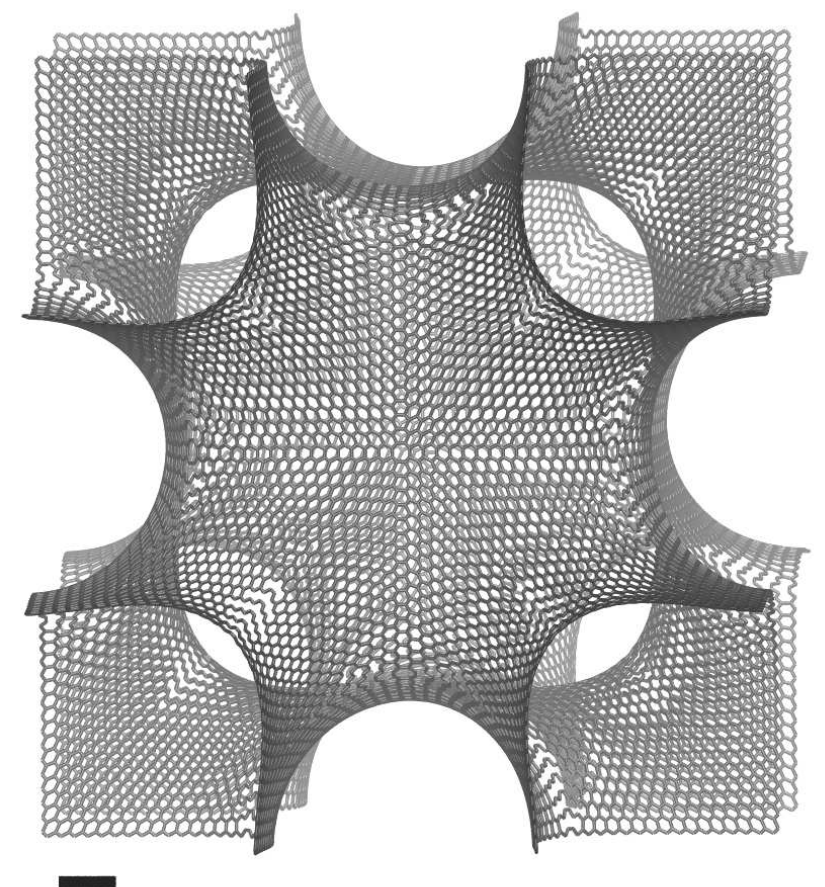

$\mathrm{b}$

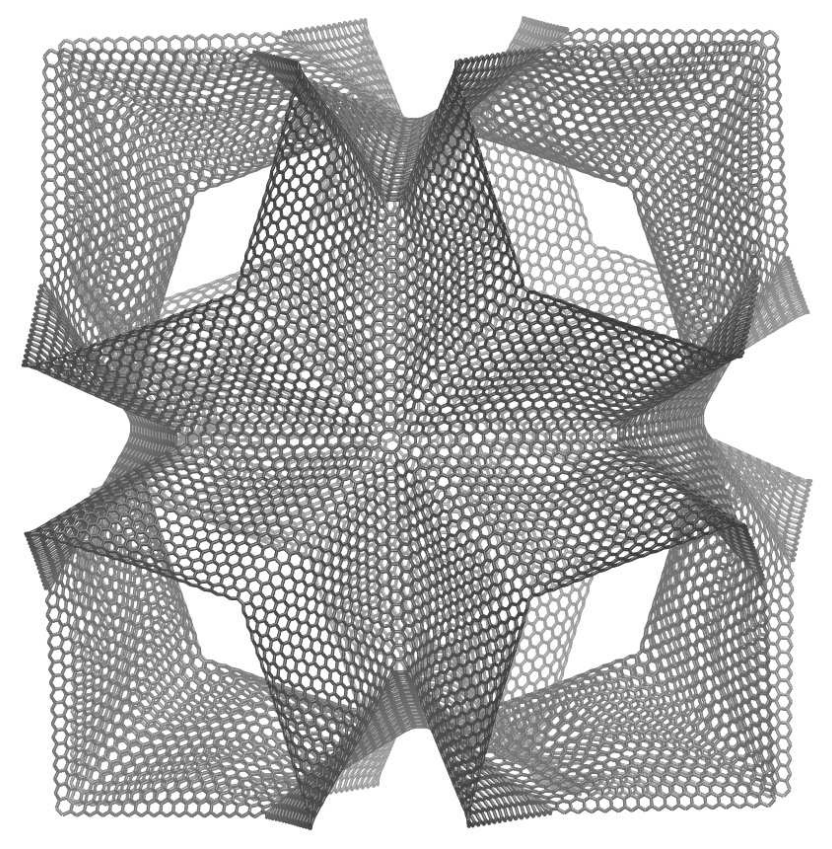

Figure 6: A single cubic cell of the I-WP8-15 structure (a) before relaxation and (b) after relaxation. Here, there are quadrilateral regions that become flat and graphenic. The scale bar in the lower left corner is for $10 \AA$. 
inverse of the elasticity tensor with values: $s_{11}, s_{12}$, and $s_{44}$. In 1940 Max Born established the conditions for elastic stability in cubic lattices as listed in Eq. 2 [20, 21].

Given the cubic elastic constants and the corresponding values from the compliance tensor, average values for Young's modulus and Poisson's ratio were calculated. These values refer to the macroscopic effective value for each quantity. Equations 3 and 4 provide the Voight average for Young's modulus and Poisson's ratio [22]. This value is at the upper bound for Young's modulus and a lower bound for the Poisson's ratio. Equations 5 and 6 give the Reuss average for these two quantities. In this case, the value is a lower bound for Young's modulus and an upper bound for Poisson's ratio. Equation 7 gives the Voigt-Reuss-Hill average for Young's modulus while Eq. 9 provides the Voigt-Reuss-Hill average for the Poisson's ratio, which requires calculating the VoigtReuss-Hill average for the shear modulus given in Eq. 8. These equations are all described by Toonder, Dommelen, and Baaijens [22]. Figures 7 and 8 shows the values of the Voigt-Reuss-Hill average for both quantities.

$$
\begin{aligned}
c_{11}-c_{12}>0 ; c_{11}+2 c_{12}>0 ; c_{44}>0 \\
E_{V}=\frac{\left(c_{11}-c_{12}+3 c_{44}\right)\left(c_{11}+2 c_{12}\right)}{c_{11}+3{ }_{12}+c_{44}} \\
\nu_{V}=\frac{c_{11}+4 c_{12}-2 c_{44}}{4 c_{11}+6 c_{12}+2 c_{44}} \\
E_{R}=\frac{5}{3 s_{11}+2 s_{12}+s_{44}} \\
\nu_{R}=-\frac{2 s_{11}+8 s_{12}-s_{44}}{6 s_{11}+4 s 12+2 s_{44}} \\
E_{V R H}=\frac{E_{V}+E_{R}}{2} \\
G_{V R H}=\frac{1}{2}\left[\frac{c_{11}-c_{12}+3 c_{44}}{5}+\frac{5}{4 s_{11}-4 s_{12}+3 s_{44}}\right] \\
\nu_{V R H}=\frac{E_{V R H}}{2 G_{V R H}}-1
\end{aligned}
$$

In addition to the average values of Young's modulus and Poisson's ratio calculated from the elastic constants, these quantities were calculated in the [100] direction of the cubic cell. These 
Average macroscopic Poisson's ratio for Schwarizites

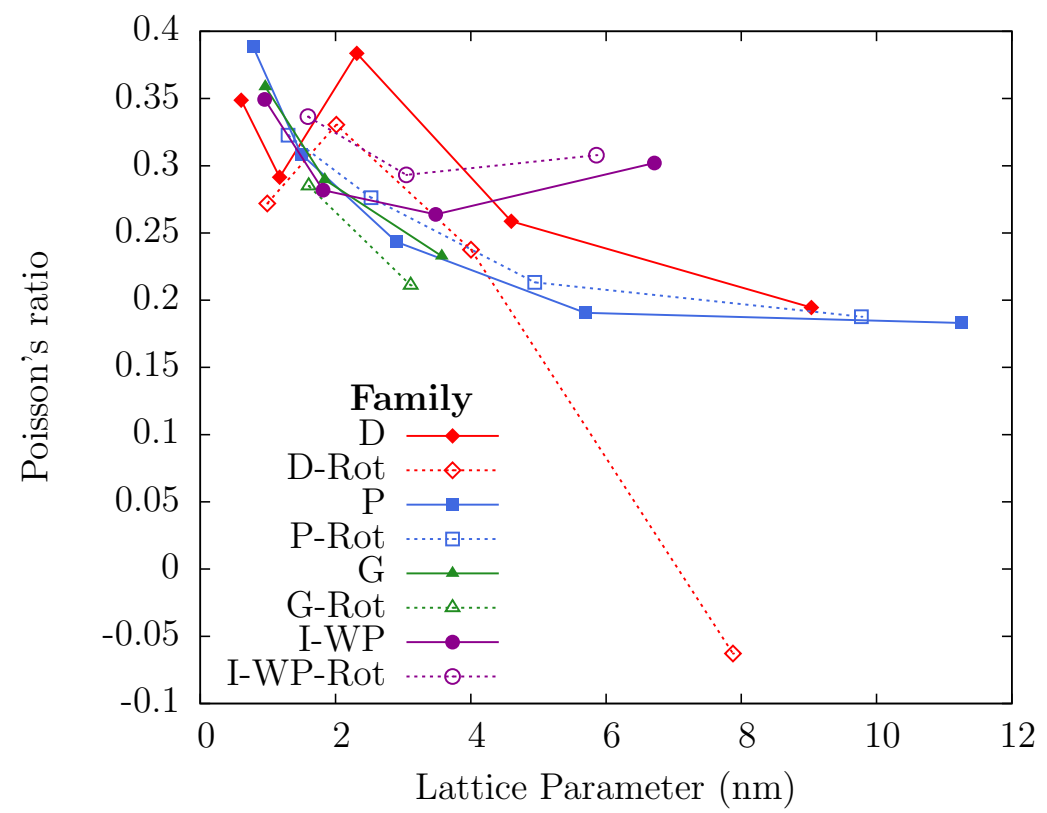

Figure 7: Averaged Poisson's ratio of graphene foams as the size of the cubic cell grows. The calculated average Poisson ratio can increase or decrease for each Schwarzite. The D8-23Rot displays a negative Poisson ratio.

Average Macroscopic Young's modulus for Schwarizites

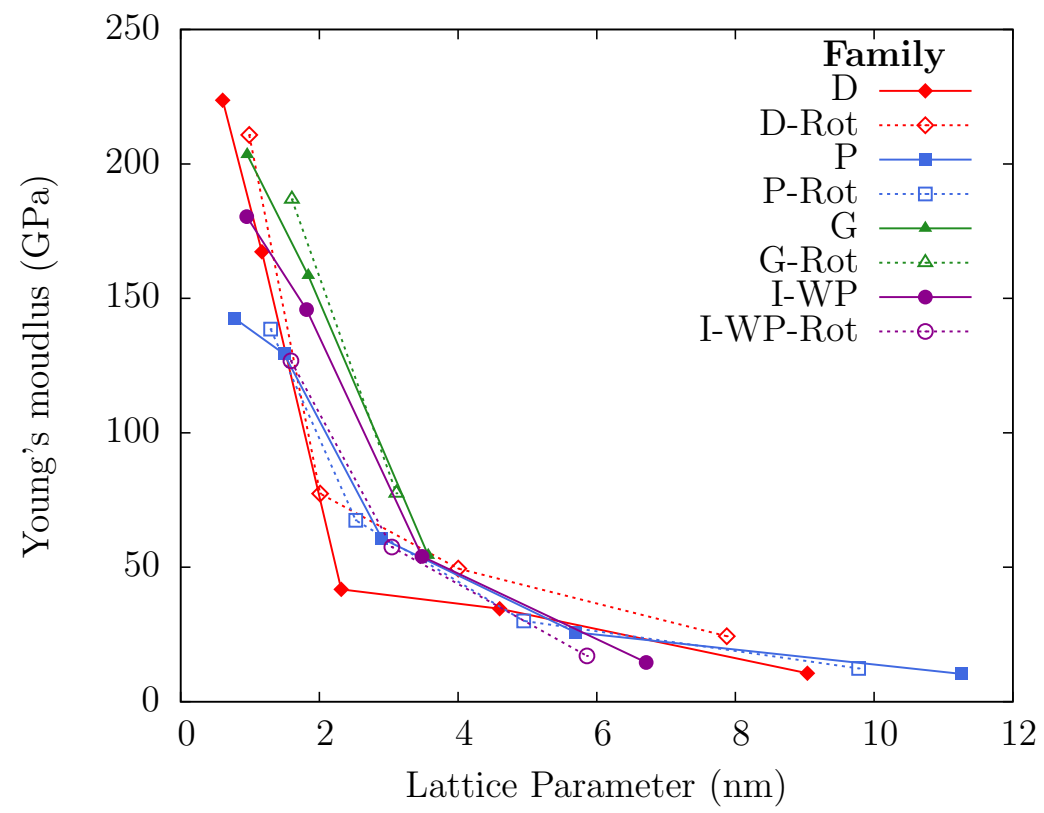

Figure 8: Averaged Young's modulus of graphene foams as the size of the cubic cell grows. The averaged Young's modulus ranges from $358 \mathrm{GPa}$ (I-WP8-0) to $5.96 \mathrm{GPa}$ (P8-15). 
values can be found in Table 2. The $\mathrm{P}$ family displays the highest values for the Poisson's ratio ranging from 0.408 to 0.353 along the direction [100]. However, for the same structures from the P family, the averaged value for the Poisson's ratio of the entire cubic cell is smaller for all cases, so that the P8-15 displays an average vale of 0.183 (see Fig. 7).

In contrast to the P family, the I-WP family displays the smallest values of the Poisson's ratio calculated in the [100] direction. However, for this family the average values of the Poisson's ratio is always larger than the Poisson's ratio along the [100] direction. Consequently, the averaged values for the I-WP family are typically similar or larger than those for the P family. In addition, for larger structures in the I-WP family the value of the Poisson's ratio increases as the lattice parameter increases (See Fig. 7). It is worth mentioning here that the D Schwarzites with ripples D8-15 and D8-23Rot possess average Poisson ratios of 0.195 and -0.063, respectively (see Figs. 9, 10 and Table 2). Note the negative value for the D8-23Rot case, thus ripples might play a role in contributing to lower the Poisson ratio of graphenic structures.

Table 1 does not include the G8-7 and the G8-11Rot Schwarzites, since these structures do not possess cubic symmetries after the relaxation since the cubic symmetry needs to be broken to find a more stable system (See Fig. 11). Also, for the I-WP8-15 and the I-WP8-23Rot, the primitive cell was used for the calculations, not the cubic cell, due to the large number of atoms present in the cubic cell (the primitive cell of a body centered cubic cell possesses half of the atoms of the cubic cell). For these structures the values for elastic properties are given in Table 3.

\subsection{Electronic Structure of the I-WP}

The electronic structure for small cases from the non-rotated genus three P, D, and G families has been studied [4]. However, there has not been work done on structures from the I-WP family which exhibits genus 4 per primitive cell. Here, we examined using DFT the I-WP8-0 (96 atoms per cubic cell), the I-WP8-1 (384 atoms per cubic cell), and the I-WP8-2Rot (288 atoms per cubic cell) Schwarzites. The I-WP8-0 Schwarzite is unique in the sense that it does not contain any hexagonal rings of carbon in its smallest case, while preserving the $\mathrm{sp}^{2}$ hybridized bonding possessing only octagonal rings (see Fig. 12). This structure displays a density of states showing

a semi-metallic behavior that resembles graphene (see Fig. 13). The calculations reveal that the 


\begin{tabular}{|l|c|c|c|c|c|c|c|}
\hline Structure & $\mathrm{n}$ & $\mathrm{a}$ & $\rho$ & $c_{11}$ & $c_{12}$ & $c_{44}$ & $K$ \\
\hline D8-0 & 24 & 6.063 & 2.147 & 313.89 & 215.51 & 117.66 & 248.31 \\
\hline D8-1 & 96 & 11.724 & 1.187 & 220.68 & 90.35 & 64.54 & 133.80 \\
\hline D8-3 & 384 & 23.148 & 0.617 & 99.78 & 41.57 & 9.40 & 60.97 \\
\hline D8-7 & 1536 & 45.990 & 0.314 & 42.06 & 14.78 & 13.79 & 23.87 \\
\hline D8-15 & 6144 & 90.370 & 0.166 & 10.16 & 3.59 & 5.41 & 5.78 \\
\hline D8-2Rot & 72 & 9.900 & 1.479 & 267.80 & 97.21 & 81.28 & 154.07 \\
\hline D8-5Rot & 288 & 20.110 & 0.705 & 129.77 & 49.41 & 23.37 & 76.20 \\
\hline D8-11Rot & 1152 & 40.030 & 0.358 & 54.33 & 20.01 & 22.16 & 31.45 \\
\hline D8-23Rot & 4608 & 78.760 & 0.188 & 55.54 & -16.03 & 5.51 & 7.82 \\
\hline P8-0 & 48 & 7.800 & 2.017 & 268.92 & 185.64 & 59.02 & 213.40 \\
\hline P8-1 & 192 & 14.911 & 1.155 & 147.31 & 99.32 & 79.83 & 115.32 \\
\hline P8-3 & 768 & 29.020 & 0.626 & 54.74 & 34.38 & 43.24 & 41.17 \\
\hline P8-7 & 3072 & 56.920 & 0.332 & 20.50 & 11.52 & 19.27 & 14.51 \\
\hline P8-15 & 12288 & 112.570 & 0.171 & 8.13 & 4.44 & 7.71 & 5.67 \\
\hline P8-2Rot & 144 & 12.984 & 1.311 & 168.87 & 114.10 & 80.70 & 132.36 \\
\hline P8-5Rot & 576 & 25.251 & 0.713 & 67.82 & 43.90 & 44.59 & 51.88 \\
\hline P8-11Rot & 2304 & 49.463 & 0.379 & 25.15 & 14.65 & 21.67 & 18.15 \\
\hline P8-23Rot & 9216 & 97.767 & 0.197 & 9.80 & 5.40 & 9.16 & 6.87 \\
\hline G8-0 & 96 & 9.601 & 2.163 & 315.10 & 202.80 & 90.77 & 240.24 \\
\hline G8-1 & 384 & 18.411 & 1.227 & 195.16 & 90.73 & 68.49 & 125.54 \\
\hline G8-3 & 1536 & 35.711 & 0.672 & 58.63 & 21.56 & 24.77 & 33.92 \\
\hline G8-7 b & 6144 & 69.331 & 0.367 & -32.32 & 26.10 & -23.49 & 6.63 \\
\hline G8-2Rot & 288 & 16.030 & 1.394 & 219.83 & 107.42 & 86.39 & 144.89 \\
\hline G8-5Rot & 1152 & 31.110 & 0.762 & 81.74 & 26.19 & 35.17 & 44.70 \\
\hline I8-WP-0 & 96 & 9.528 & 2.212 & 404.94 & 109.36 & 36.75 & 207.89 \\
\hline I8-WP-1 & 384 & 18.148 & 1.281 & 235.75 & 50.20 & 40.55 & 112.05 \\
\hline I8-WP-3 & 1536 & 34.800 & 0.726 & 84.29 & 15.33 & 15.37 & 38.32 \\
\hline I8-WP-7 & 6144 & 67.140 & 0.404 & 25.79 & 5.73 & 3.71 & 12.42 \\
\hline I8-WP-2Rot & 288 & 15.904 & 1.427 & 253.60 & 70.76 & 29.56 & 131.71 \\
\hline I8-WP-5Rot & 1152 & 30.460 & 0.812 & 95.39 & 22.23 & 15.74 & 46.62 \\
\hline I8-WP-11Rot & 4608 & 58.590 & 0.456 & 31.71 & 6.72 & 4.08 & 15.05 \\
\hline
\end{tabular}

Table 1: Table of the calculated mechanical properties for all cubic cases of Schwarzites examined in this paper. The number of atoms per cubic cell (n) is given in addition to the lattice parameter (a) given in angstroms. The density $(\rho)$ is given in $\mathrm{g} / \mathrm{cm}^{3}$. The elastic constants $\left(c_{11}, c_{12}, \& c_{44}\right)$, the bulk modulus $(K)$, and Young's modulus $\left(Y_{100}\right)$, given perpendicular to the cubic faces, are given in GPa. Lastly, is the Poisson's ratio $\left(\nu_{100}\right)$, given perpendicular to the cubic faces.

aThese structures exhibits ripples when relaxed

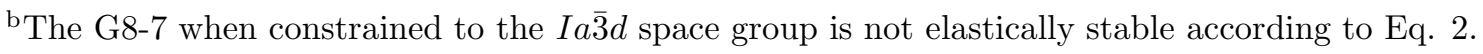




\begin{tabular}{|l|c|c|c|c|}
\hline Structure & $Y_{100}$ & $Y_{\text {avg }}$ & $\nu_{100}$ & $\nu_{\text {avg }}$ \\
\hline D8-0 & 138.434 & 223.699 & 0.407 & 0.349 \\
\hline D8-1 & 168.188 & 167.346 & 0.290 & 0.292 \\
\hline D8-3 & 75.331 & 41.749 & 0.294 & 0.384 \\
\hline D8-7 & 34.373 & 34.564 & 0.260 & 0.259 \\
\hline D8-15 & 8.291 & 10.586 & 0.260 & 0.195 \\
\hline D8-2Rot & 216.028 & 210.811 & 0.266 & 0.272 \\
\hline D8-5Rot & 102.529 & 77.392 & 0.275 & 0.331 \\
\hline D8-11Rot & 43.566 & 49.520 & 0.269 & 0.238 \\
\hline D8-23Rot ${ }^{\text {a }}$ & 42.527 & 24.315 & -0.405 & -0.063 \\
\hline P8-0 & 117.291 & 142.535 & 0.408 & 0.389 \\
\hline P8-1 & 67.321 & 129.354 & 0.402 & 0.309 \\
\hline P8-3 & 28.226 & 60.715 & 0.385 & 0.243 \\
\hline P8-7 & 12.214 & 25.811 & 0.359 & 0.191 \\
\hline P8-15 & 4.997 & 10.368 & 0.353 & 0.183 \\
\hline P8-2Rot & 76.866 & 138.557 & 0.403 & 0.323 \\
\hline P8-5Rot & 33.315 & 67.461 & 0.392 & 0.276 \\
\hline P8-11Rot & 14.376 & 30.015 & 0.367 & 0.213 \\
\hline P8-23Rot & 5.960 & 12.371 & 0.355 & 0.188 \\
\hline G8-0 & 156.281 & 203.455 & 0.391 & 0.359 \\
\hline G8-1 & 137.580 & 158.476 & 0.317 & 0.290 \\
\hline G8-3 & 47.035 & 54.380 & 0.268 & 0.233 \\
\hline G8-2Rot & 149.300 & 186.870 & 0.328 & 0.285 \\
\hline G8-5Rot & 69.030 & 77.504 & 0.242 & 0.211 \\
\hline I8-WP-0 & 358.420 & 180.415 & 0.212 & 0.349 \\
\hline I8-WP-1 & 218.120 & 145.844 & 0.175 & 0.282 \\
\hline I8-WP-3 & 79.570 & 54.042 & 0.153 & 0.264 \\
\hline I8-WP-7 & 23.710 & 14.581 & 0.181 & 0.302 \\
\hline I8-WP-2Rot & 222.720 & 126.753 & 0.218 & 0.337 \\
\hline I8-WP-5Rot & 86.980 & 57.498 & 0.189 & 0.293 \\
\hline I8-WP-11Rot & 29.360 & 17.042 & 0.175 & 0.308 \\
\hline
\end{tabular}

Table 2: Table of Young's moduli and Poisson's ratios for all elastically stable, cubic cases of Schwarzites examined in this paper. The Young's modulus $(Y)$, given in GPa, is listed perpendicular to the cubic faces (in the [100] direction) or as the macroscopic averaged values. The Poisson's ratio $(\nu)$ is also given perpendicular to the cubic faces and as the macroscopic averaged value.

${ }^{a}$ These structures exhibits ripples when relaxed 


\begin{tabular}{|l|c|c|c|c|c|c|c|c|c|c|c|c|}
\hline Structure & $\mathrm{a}$ & $\rho$ & $\mathrm{K}$ & $\mathrm{Y}_{x}$ & $\mathrm{Y}_{y}$ & $\mathrm{Y}_{z}$ & $\nu_{x y}$ & $\nu_{x z}$ & $\nu_{y x}$ & $\nu_{y z}$ & $\nu_{z x}$ & $\nu_{z y}$ \\
\hline G8-7 & $a=67.35$ & 0.39 & 4.52 & 8.24 & 23.00 & 8.50 & 0.230 & -0.050 & 0.080 & 0.040 & 0.050 & 0.127 \\
& $b=70.24$ & & & & & & & & & & \\
& $c=67.10$ & & & & & & & & & & \\
& $\alpha=90.71$ \\
& $\beta=89.00$ & & & & & & & & & & & \\
& $\gamma=90.64$ & & & & & & & & & & & \\
\hline G8-11Rot & $a=58.95$ & 0.44 & 5.62 & 8.30 & 23.32 & 8.18 & 0.140 & 0.260 & 0.050 & 0.050 & 0.270 & 0.160 \\
& $b=60.76$ & & & & & & & & & & & \\
& $c=58.95$ & & & & & & & & & & & \\
& $\alpha=89.51$ & & & & & & & & & & & \\
\hline I-WP8-15 & $\gamma=91.08$ & & & & & & & & & & & \\
\hline I-WP8-23Rot & 114.03 & 0.25 & 4.46 & 2.84 & 3.32 & 3.32 & 0.458 & 0.458 & 0.393 & 0.293 & 0.393 & 0.293 \\
\hline
\end{tabular}

Table 3: Table of the calculated mechanical properties for all special cases of Schwarzites examined in this paper. This includes cases without cubic symmetry or cases where the primitive cell was used as a result of the large number of atoms in the cubic cell. The lattice parameter for non-cubic cases is given for all three dimensions and includes the angles of the cell. In all cases, the lattice parameter is given in angstroms while the angles are given in degrees. The density $(\rho)$ is given in $\mathrm{g} / \mathrm{cm}^{3}$. The bulk modulus $(\mathrm{K})$ and the Young's modulus, given in three directions $\left(\mathrm{Y}_{x}\right.$, $\mathrm{Y}_{y}$, and $\mathrm{Y}_{z}$ ), are all given in GPa. Lastly, Poisson's ratio $(\nu)$ is given with two indices, the first referring to the direction of stress being applied and the second referring to the direction of expansion or contraction. 
a

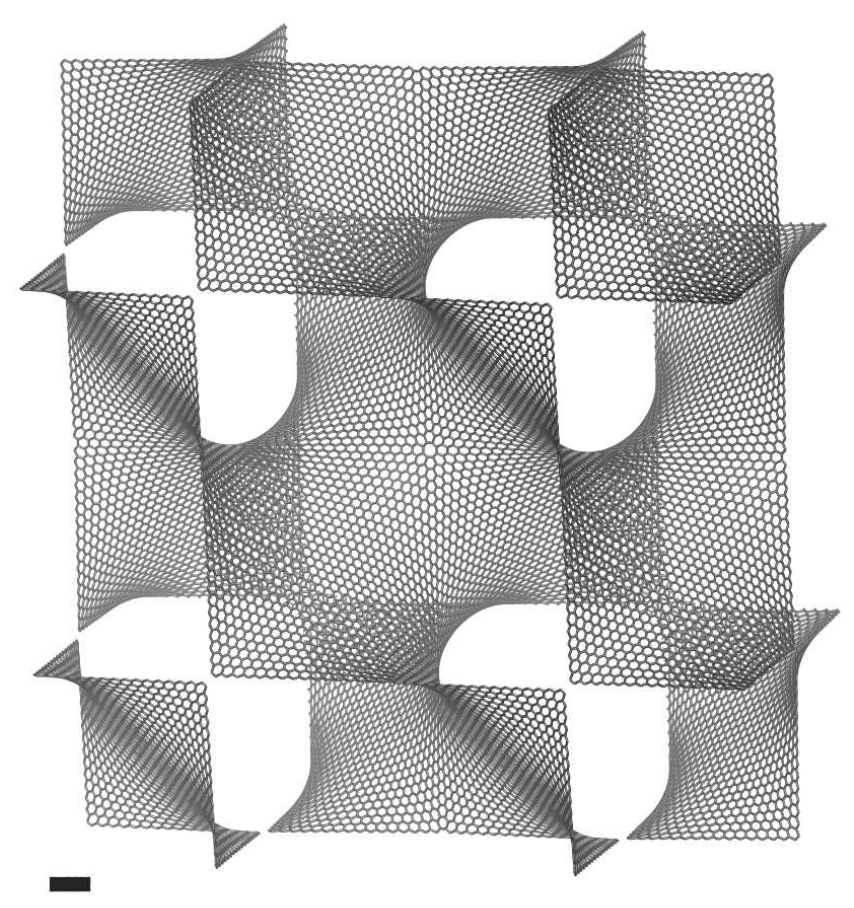

b

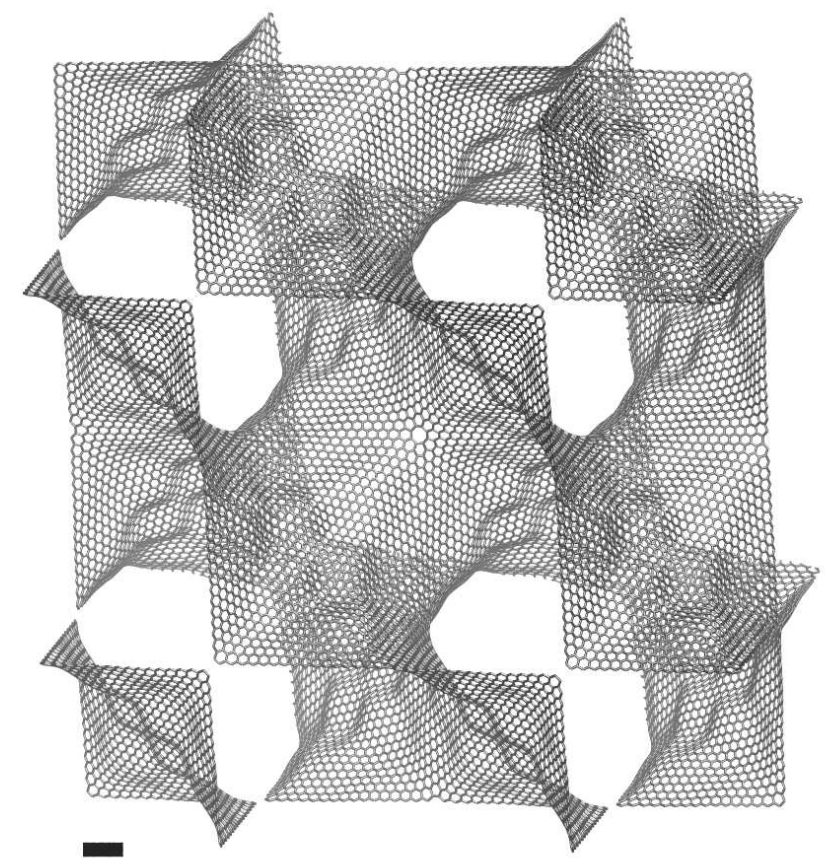

Figure 9: The front half of the cubic cell of the D8-15 structure (a) before relaxation and (b) after relaxation. The scale bar in the lower left corner is for $10 \AA$.

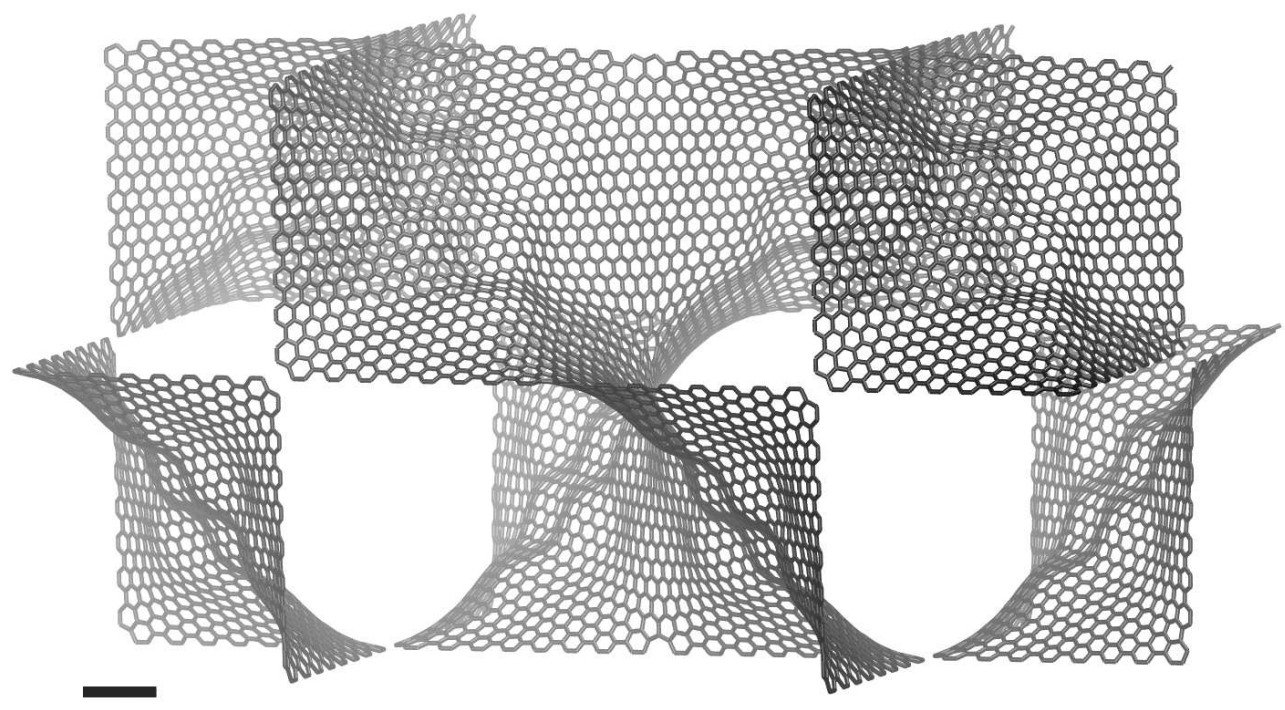

Figure 10: Two cubic cells of the D8-23Rot structure. This variation of the structure displays ripples in the hexagonal regions with lower curvature. While this structure has slightly higher energetics when compared to the relaxation without ripples (see Fig. 4) the averaged Poisson's ratio is predicted to be negative (see Fig. 7). The octagonal rings that lie on the boundary of the surface are not complete as some of the atoms lie in the next cubic cell. The scale bar in the lower left corner is for $10 \AA$. 
a

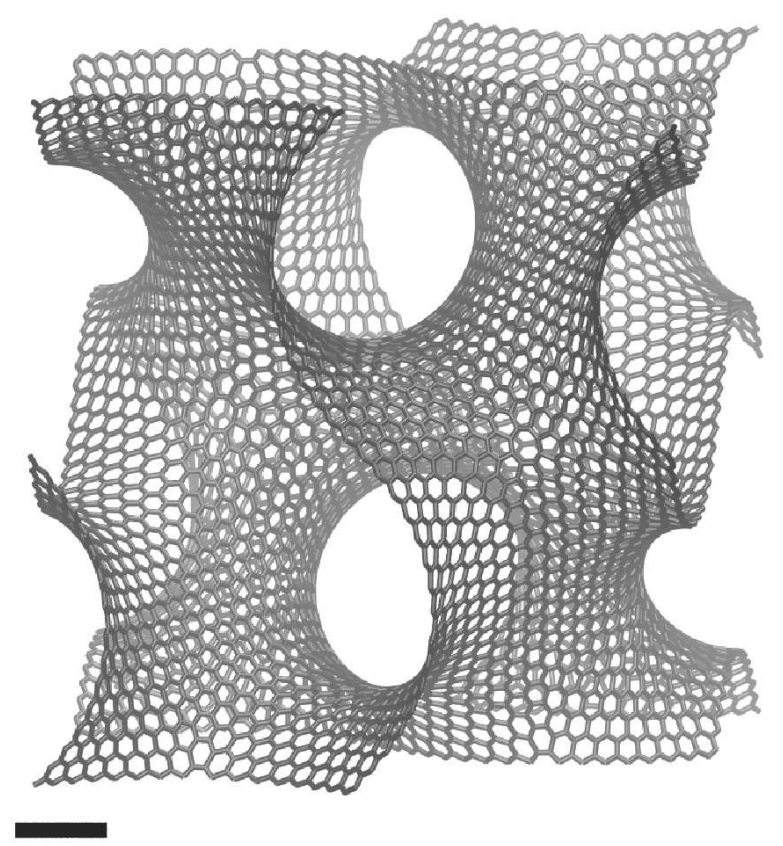

$\mathrm{b}$

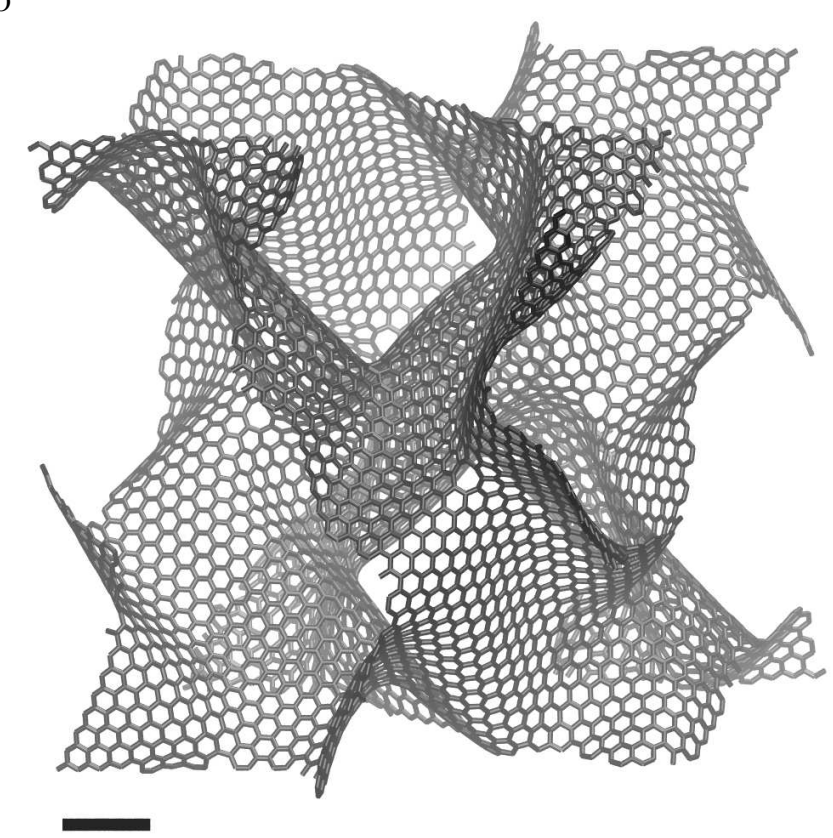

Figure 11: A single cubic cell of the G8-7 structure (a) before relaxation compared to (b) after relaxation without constraint to the $I a \overline{3} d$ space group. This structure still contain a symmetric point of inversion in the center. It can be seen that the structure becomes distorted as small regions become more graphenic when relaxed. This structure is elastically stable unlike the other relaxation of the G8-7 and also has slightly lower energetics in comparison (see Fig. 4). The scale bar in the lower left corner is for $10 \AA$. 


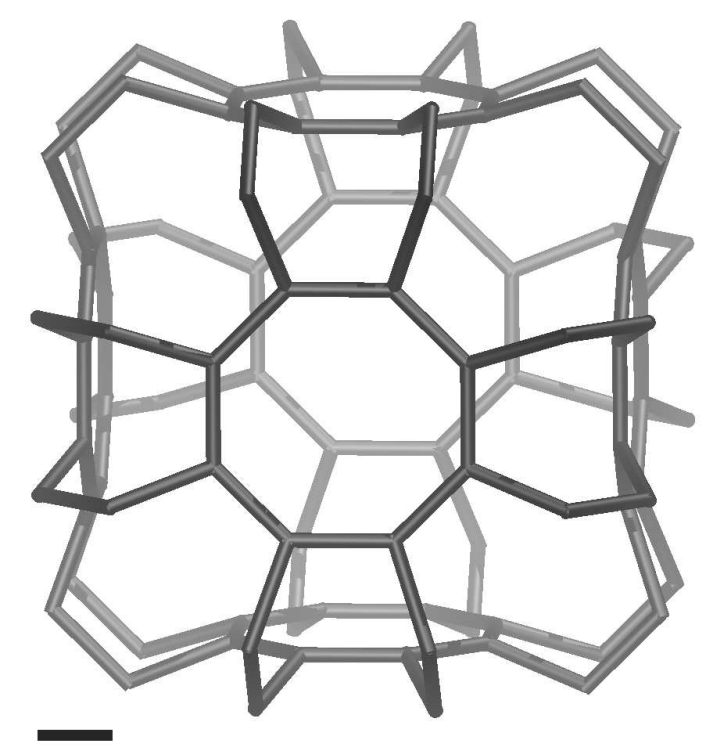

Figure 12: A single cubic cell of the relaxed IWP8-0 structure. This structure only contains octagonal rings. The octagonal rings parallel to the cubic faces appear flat while the octagonal rings along regions of higher curvature are more distorted. The scale bar in the bottom left represents $1 \AA$.

slightly larger I-WP8-1 Schwarzite is a semiconductor with an indirect band gap calculated, to be $0.866 \mathrm{eV}$, and the I-WP8-2Rot, which lies in size between the I-WP8-0 and the I-WP8-1, exhibits metallic behavior. From these results we conclude, that the electronic properties involve a variety of behaviors, depending on the size. What remains an open question is if by increasing the size, hyper Dirac cones can be found as in the case of one the G Schwarzites [12].

\section{Conclusion}

The mechanical properties of new giant Schwarzites have been calculated in order to shed light on the observed properties of the experimentally synthesized graphene foams. Regarding the stability of these periodic $\mathrm{sp}^{2}$ carbons, we have gone further than just considering the energetics, and also consider the elastic stability by satisfying the Born criteria.

For all the elastically stable cases studied, the Young modulus decreases as the size of the Schwarzite increases. Poisson's ratios calculated are not as as low as those found by Wu, Y. et. al. in experimentally produced graphene foams from graphene oxide [5]. We believe that this might be due to the defects present in graphene oxide. In fact, one of the models used by Wu, Y. et. al. is similar to one of the the I-WP cases studied here, but containing defects such as pentagonal and additional octagonal rings. Thus, further work should examine the introduction of topological 
$\mathrm{a}$

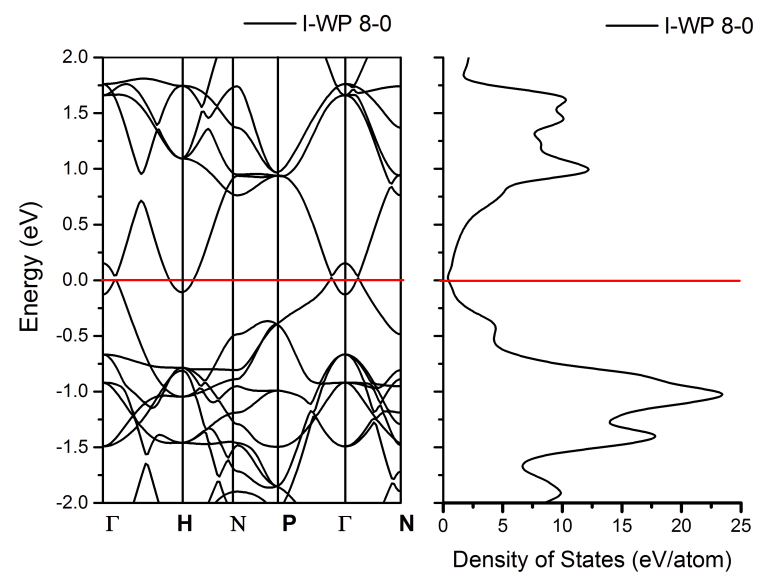

$\mathrm{C}$

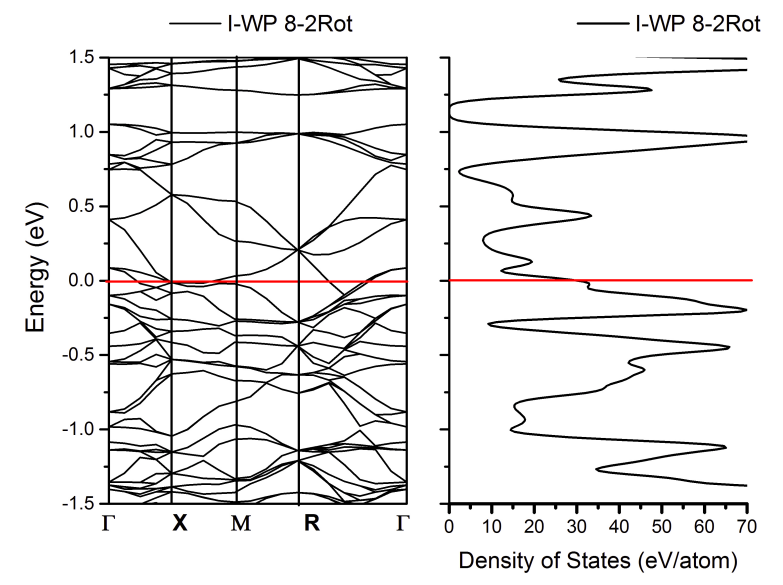

$\mathrm{b}$

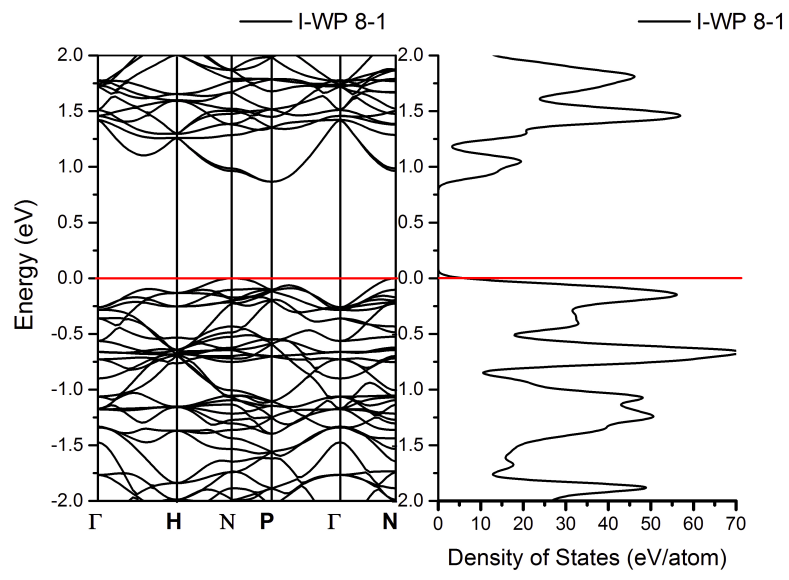

Figure 13: Band structure and density of states for (a) the I-WP8-0, (b) the I-WP8-1 (top right), and (c) the I-WP8-2Rot (bottom center) Schwarzites. For the I-WP8-0 there are multiple locations of crossing at the Fermi energy near the $\Gamma$ and $H$ points in the Brillouin zone. The density of states appears similar to that of single layer graphene. The I-WP8-1 structure has an indirect gap of $0.866 \mathrm{eV}$ near the $P$ and $N$ points in the Brillouin zone. Lastly, the I-WP8-2Rot appears to be metallic from the density of states. 
defects in these giant Schwarzites. In addition, from our results we found that as the structures grows, ripples are formed that can also form re-entrant surfaces that could cause a decrease in the Poison's ratio. We showed that for the giant D Schwarzite with ripples, the Poisson ratio decreases and becomes negative. Negative Poisson ration materials out of graphene constitute a fascinating possibility that needs further experimental studies.

Defects also play an important role in the mechanical and electronic properties of graphene. Two primary defects have been previously considered, Stone-Thrower-Wales (STW) defects, involving a bond rotation to create two heptagonal and two pentagonal rings, and divacancy defects, the removal of two atoms to form two pentagonal rings and an octagonal ring [8]. Carlsson and Scheffler have shown that in small concentrations STW defects are stable [23]. Grima et. al. found that introducing divacancy defects onto single layer graphene results in a negative Poisson's ratio [24]. In this contex, Dettori, Cadelano, and Colombo have shown a decrease in the Poisson's ratio through the introduction of STW defects in graphene [25].

Another result we found is that as the gyroid Schwarzites grow around 6144 atoms per cubic cell, the structures become elastically unstable and in order to stabilize them the symmetry needs to be broken: We believe this happens because the gyroid-type TPMS do not exhibit straight lines as graphene does, so there is a competition between the flatness of graphene and the symmetry of the surface. Therefore, when the structure becomes larger, it becomes more graphenic (flat) and the symmetry needs to be broken to be stable, thus producing structures with very low Poisson's ratios close to zero along its faces (see Table 3). From an energetics standpoint, the geometry, and from the average values of the Poisson's ratio, we conclude that the most promising giant Schwarzite to be synthesized is the P-type since it is composed of hexagonal graphene flakes which can be stitched together by a self assembly technique involving edge functionalization. The other cases would require a template such as a mesoporous silicate or an opal type structure [26]. We calculated using DFT, the electronic properties of I-WP Schwarzites, not previously analysed before, finding that they exhibit semiconducting, semimetallic or metallic behavior depending on their size. An interesting point that needs further study is the possibility of finding Dirac cones in giant Schwarzites since these are more graphenic than previously studied cases in which the ratios 
of hexagons to octagons is very large.

\section{Acknowledgements}

H. T. acknowledges the support from the National Science Foundation (EFRI-1433311). M.T. acknowledges support by the U.S. Air Force Office of Scientific Research MURI grant FA955012-1-0035, by the Materials Simulation Center of the Materials Research Institute (Penn State),

the Research Computing and Cyberinfrastructure unit of Information Technology Services and Penn-State Center for Nanoscale Science, the Penn State Center for Nanoscale Science for seed grant on 2-D Layered Materials (DMR-0820404). The authors also acknowledge the Center for 2-Dimensional and Layered Materials at the Pennsylvania State University.

\section{References}

[1] Mackay AL, Terrones H. Diamond from graphite. Nature 1991;352(6338):762-762. doi:10.1038/352762a0.

[2] Terrones H, Mackay A. The geometry of hypothetical curved graphite structures. Carbon 1992;30(8):1251-1260. doi:10.1016/0008-6223(92)90066-6.

[3] Terrones H, Terrones M. Curved nanostructured materials. New Journal of Physics 2003;5. doi: $\{10.1088 / 1367-2630 / 5 / 1 / 126\}$.

[4] Huang MZ, Ching W, Lenosky T. Electronic properties of negative-curvature periodic graphitic carbon surfaces. Physical Review B 1993;47(3):1593-1606. doi:10.1103/physrevb.47.1593.

[5] Wu Y, Yi N, Huang L, Zhang T, Fang S, Chang H, et al. Three-dimensionally bonded spongy graphene material with super compressive elasticity and near-zero poisson's ratio. Nature Communications 2015;6:6141. doi:10.1038/ncomms7141.

[6] Schwarz H. Gesammelte Mathematische Abhandlungen; vol. 1. Verlag J. Springer Berlin; 1890. 
[7] Schoen AH. Infinite periodic minimal surfaces without self-intersections. Tech. Rep.; NASA Technical Note D-5541; 1970.

[8] Lv R, Cruz-Silva E, Terrones M. Building complex hybrid carbon architectures by covalent interconnections: Graphene-nanotube hybrids and more. ACS Nano 2014;8(5):4061-4069. URL: http://dx.doi.org/10.1021/nn502426c. doi:10.1021/nn502426c.

[9] Novoselov KS, Geim AK, Morozov SV, Jiang D, Katsnelson MI, Grigorieva IV, et al. Twodimensional gas of massless dirac fermions in graphene. Nature 2005;438(7065):197-200. doi:10.1038/nature04233.

[10] Terrones M, Botello-Méndez AR, Campos-Delgado J, López-Urías F, Vega-Cantú YI, Rodríguez-Macías FJ, et al. Graphene and graphite nanoribbons: Morphology, properties, synthesis, defects and applications. Nano Today 2010;5(4):351-372. doi:10.1016/j.nantod.2010.06.010.

[11] Neto AHC, Guinea F, Peres NMR, Novoselov KS, Geim AK. The electronic properties of graphene. Reviews of Modern Physics 2009;81(1):109-162. doi:10.1103/revmodphys.81.109.

[12] Lherbier A, Terrones H, Charlier JC. Three-dimensional massless dirac fermions in carbon schwarzites. Physical Review B 2014;90(12). doi:10.1103/physrevb.90.125434.

[13] Brakke KA. The surface evolver. Experimental Mathematics 1992;1(2):141-165. doi:10.1080/10586458.1992.10504253.

[14] Brenner DW, Shenderova OA, Harrison JA, Stuart SJ, Ni B, Sinnott SB. A second-generation reactive empirical bond order (REBO) potential energy expression for hydrocarbons. Journal of Physics: Condensed Matter 2002;14(4):783-802. doi:10.1088/0953-8984/14/4/312.

[15] Gale JD, Rohl AL. The general utility lattice program ( GULP ). Molecular Simulation 2003;29(5):291-341. doi:10.1080/0892702031000104887.

[16] Clark SJ, Segall MD, Pickard CJ, Hasnip PJ, Probert MIJ, Refson K, et al. First princi- 
ples methods using CASTEP. Zeitschrift für Kristallographie 2005;220(5/6/2005):567-570. doi:10.1524/zkri.220.5.567.65075.

[17] Ceperley DM, Alder BJ. Ground state of the electron gas by a stochastic method. Phys Rev Lett 1980;45(7):566-569. doi:10.1103/physrevlett.45.566.

[18] Perdew JP, Zunger A. Self-interaction correction to density-functional approximations for many-electron systems. Physical Review B 1981;23(10):5048-5079. doi:10.1103/physrevb.23.5048.

[19] Valencia F, Romero AH, Hernández E, Terrones M, Terrones H. Theoretical characterization of several models of nanoporous carbon. New J Phys 2003;5:123-123. doi:10.1088/1367-2630/5/1/123.

[20] Born M. On the stability of crystal lattices. i. Mathematical Proceedings of the Cambridge Philosophical Society 1940;36:160-172. doi:10.1017/S0305004100017138.

[21] Mouhat F, Coudert FX. Necessary and sufficient elastic stability conditions in various crystal systems. Physical Review B 2014;90(22). doi:10.1103/physrevb.90.224104.

[22] den Toonder JMJ, van Dommelen JAW, Baaijens FPT. The relation between single crystal elasticity and the effective elastic behaviour of polycrystalline materials: theory, measurement and computation. Modelling and Simulation in Materials Science and Engineering 1999;7(6):909-928. doi:10.1088/0965-0393/7/6/301.

[23] Carlsson JM, Scheffler M. Structural, electronic, and chemcial properties of nanoporous carbon. Physical Review Letters 2006;96:046806. doi:10.1103/PhysRevLett.96.046806.

[24] Grima JN, Winczewski S, Mizzi L, Grech MC, Gatt RR, Attard D, et al. Tailoring graphene to achieve negative poisson's ratio properties. Advanced Materials 2015;27:14551459. doi:10.1002/adma. 201404106.

[25] Dettori R, Cadelano E, Colombo L. Elastic fields and moduli in defected graphene. Journal of Physics: Condensed Matter 2012;24(10):104020. doi:10.1088/0953-8984/24/10/104020. 
[26] Zakhidov AA. Carbon structures with three-dimensional periodicity at optical wavelengths. Science 1998;282(5390):897-901. doi:10.1126/science.282.5390.897. 